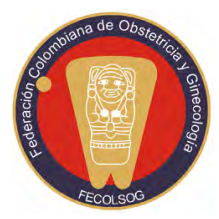

Revista Colombiana de Obstetricia y Ginecología Vol. 71 No. 3 •Julio-Septiembre 2020 • (265-274)

\title{
TRASPLANTE ORTOTÓPICO DE ÚTERO. EXPERIMENTO EN MODELO OVINO, CALI (COLOMBIA)
}

\section{Orthotopic uiterus transplantation. Sheep model experiment, Calli (Colombia)}

Felipe Castro-Villegas, $M D^{1}$; Gustavo Adolfo Canaval-Erazo, $M D^{2}$; Juan Manuel Rico-Juri, MD' ${ }^{1}$; José Óscar Gutiérrez-Montes, $M D, M S c^{3}$; Anabel Vanin-Aguas, $M^{1}$; Hoover Orlando Canaval-Erazo, $M D^{2,4}$

Recibido: 11 de marzo de 2020/Aceptado: 03 de agosto de 2020

\section{RESUMEN}

Objetivo: se ha recomendado a los profesionales que tengan como proyecto realizar trasplante uterino, hacer previamente trabajos experimentales en animales. Este trabajo describe el procedimiento del trasplante uterino en ovejas y los resultados a corto y mediano plazo.

Materiales y métodos: estudio de cirugía experimental en ovejas sometidas a explante y trasplante uterino. A cuatro ovejas de 40-50 kg de peso les fue trasplantado el útero (ortotópico) de cuatro ovejas vivas donantes. Se utilizó técnica de anastomosis vascular término-lateral, la vagina se suturó en un plano y el útero se fijó a la pared pélvica. Se describen las complicaciones y la evolución a 180 días. Resultados: se realizó la cirugía de trasplante en las cuatro ovejas. El tiempo quirúrgico fue de 240

* Correspondencia: Hoover Canaval-Erazo, Cra. 39A \# 5A-100 Cali. hoover.canaval@correounivalle.edu.co

1 Médico cirujano, especialista en trasplante hepático, Centro Médico Imbanaco, Cali (Colombia).

2 Médico ginecobstetra, Centro Médico Imbanaco, Cali (Colombia).

3 Profesor de Farmacología y Medicina Regenerativa, Escuela de Medicina, Facultad de Salud, Universidad del Valle, Cali (Colombia)

4 Médico ginecobstetra, Centro Médico Imbanaco, Cali (Colombia). Profesor del Departamento de Ginecología y Obstetricia, Escuela de Medicina, Facultad de Salud, Universidad del Valle, Cali (Colombia). minutos (min) en el primer procedimiento y de 185 min en el último, y el tiempo de isquemia caliente se redujo de 42 a $22 \mathrm{~min}$. Una oveja murió al séptimo día posoperatorio por una complicación intraoperatoria ajena a las anastomosis vasculares. Otra oveja que fue trasplantada desarrolló infección-local vaginal manejada con metronidazol, con evolución satisfactoria. Las tres ovejas no habían presentado rechazo al trasplante a los 6 meses.

Conclusiones: el modelo ovino permite entrenamiento quirúrgico en cirugía experimental de trasplante uterino. Para los autores constituyó adquisición de conocimiento y avanzar hacia la realización futura del trasplante uterino en mujeres con infertilidad absoluta por factor uterino en Colombia.

Palabras clave: trasplante; útero; infertilidad.

\section{ABSTRACT}

Objective: It has been recommended that professionals who are planning to perform uterine transplantation should first carry out animal experiments. This paper describes the procedure for uterine transplant in sheep, as well as short and medium- term results. 
Materials and methods: Experimental surgery study in sheep subjected to uterine explantation and transplant. Four 40-50 kg sheep received uteri transplantation (orthotopic) from four live donors. End-to-side vascular anastomosis was used, the vagina was sutured on one plane and the uterus was fixed to the pelvic wall. Complications and 180-day evolution are described.

Results: Transplant surgery was accomplished in the 4 sheep. Surgical time in the first procedure was 240 minutes, while the last procedure lasted 185 minutes. Warm ischemia time was reduced from 42 to 22 minutes. One sheep died on the seventh postoperative day due to an intraoperative complication unrelated to the vascular anastomosis. A second sheep developed local vaginal infection treated with metronidazole and evolved satisfactorily. No transplant rejection had occurred in the remaining 3 sheep after 6 months.

Conclusions: The ovine model allowed surgical training in experimental uterine transplant surgery. For the authors, it offered an opportunity to gain knowledge and make progress towards future uterus transplantation in women with absolute uterine factor infertility in Colombia.

Key words: Transplant; uterus; infertility.

\section{INTRODUCCIÓN}

La infertilidad se define como la imposibilidad de lograr un embarazo clínico después de 12 meses o más de relaciones sexuales regulares sin protección (1). En Colombia, de acuerdo con la Encuesta Nacional de Demografía en Salud 2015 (ENDS), el porcentaje de mujeres de 13 a 49 años que desean uno o más hijos y han tenido problemas de fertilidad es de 10,2\%. Los problemas de infertilidad se concentran en el grupo de mujeres de 40 a 44 años con el 25,4\% (2). En la literatura se ha reportado una incidencia de infertilidad a nivel global del 10,5\% de la población (3).

Parte de los casos de infertilidad son producidos por alteraciones uterinas. En el proceso reproductivo, el útero participa en eventos importantes como el transporte del espermatozoide, la implantación embrionaria y la nutrición embrio-fetal; por ello, las alteraciones en el útero, sean congénitas o adquiridas, pueden tener un papel negativo en la fertilidad (4). En una revisión narrativa de la literatura, Acién informa que la frecuencia de anormalidades uterinas en población general varía entre un 2 y $4 \%$, y en mujeres con antecedente de infertilidad llega al $16 \%$ (5). Se ha descrito que en mujeres sometidas a fertilización in vitro las anormalidades uterinas llegan a un 27\% (6). Las mujeres con anomalías congénitas del útero presentan infertilidad en una frecuencia que varía entre el 3 y el $6 \%(4,7)$.

De acuerdo con The American Fertility Society (1988), se consideran siete tipos de anomalías uterinas congénitas: hipoplasia o agenesia uterina, útero unicorne, útero didelfo, útero bicorne, útero septado, útero arcuato y el expuesto a dietilestilbestrol (8). Algunas de estas malformaciones uterinas producen infertilidad absoluta por factor uterino (IAFU) o infertilidad femenina no tratable (9). Las principales causas de infertilidad no tratable son la hipoplasia uterina, el útero unicorne o bicorne y el síndrome de Mayer-Rokitansky-Küster-Hauser (MRKH). Otras causas de infertilidad no tratable son: la presencia de sinequias endometriales severas, ausencia uterina secundaria a la extirpación del órgano por miomatosis uterina gigante o por tumores malignos (9). Las opciones que tienen las mujeres con IAFU son la adopción, la madre sustituta o subrrogada y el trasplante uterino. En Colombia, en la actualidad, la única opción es la adopción (10), ya que la legislación no es clara respecto a la madre subrogada que, si bien no está claramente prohibida, se ha sugerido que se requiere un marco jurídico que la regule (11). Por otra parte, en el país no hay centros que estén realizando trasplantes uterinos.

El primer intento de trasplante uterino fue realizado en Arabia Saudita en el 2000, utilizando el útero de una mujer donante viva, pero el injerto se perdió al día 99 posoperatorio por un fenómeno de trombosis vascular del injerto, secundario a un prolapso de este (12). En 2014 nace el primer bebé 
producto de un trasplante uterino de donante viva, en un grupo de 9 mujeres sometidas a trasplantes en Gotemburgo, Suecia (13); posteriormente, en 2017 nace en São Paulo, Brasil, el primer bebé producto de un trasplante de útero obtenido de una mujer donante cadáver (14). El trasplante uterino en humanos está aún en fase experimental (9) y presenta múltiples consideraciones éticas por resolver (15).

La Federación Internacional de Ginecología y Obstetricia (FIGO), en 2009, recomienda a profesionales que tengan como proyecto realizar trasplante uterino hacer previamente trabajos experimentales en animales (16). Se han utilizado múltiples modelos animales como ratas, conejos, cerdos, ovejas y primates para entrenamiento técnico del trasplante de órganos reproductores femeninos y en algunos de ellos se han logrado embarazos (17-19). El modelo ovino, por sus similitudes anatómicas vasculares con el humano, es uno de los más utilizados en el entrenamiento para la realización del trasplante uterino (20). Los equipos más experimentados en Suecia, Brasil, Estados Unidos y República Checa realizaron su entrenamiento en ovejas (21).

El objetivo de este estudio es presentar la experiencia del entrenamiento en ovejas de un equipo de cirujanos en Colombia, con énfasis en la descripción de la preparación prequirúrgica, el procedimiento quirúrgico, el manejo de los órganos donados y el control posoperatorio; secundariamente, presentar los resultados de la cirugía en las ovejas, con el propósito de consolidar la fase inicial del entrenamiento quirúrgico, que oriente el desarrollo posterior hacia la creación de un programa de trasplante uterino en mujeres de nuestro país, además de estimular la creación de centros de entrenamiento por las posibilidades que ofrece en el futuro esta cirugía compleja.

\section{MATERIALES Y MÉTODOS}

Diseño y población. Estudio experimental en ovejas, sin grupo control. Se incluyeron ovejas con edad superior a los 1,5 años y menor a 5 años, con pesos corporales por encima de los $40 \mathrm{~kg}$, con historia de al menos un embarazo con producto vivo. Se excluyeron ovejas con alguna enfermedad infecciosa diagnosticada y vigente al momento de su selección prequirúrgica. Todas las intervenciones se llevaron a cabo entre los meses de febrero y marzo de 2018, en el Laboratorio de Cirugía Experimental de la Facultad de Salud de la Universidad del Valle, en Cali, Colombia, y contaron con la participación de personal profesional del área quirúrgica del Centro Médico Imbanaco de Cali. Las ovejas fueron intervenidas por un cirujano líder, profesional titulado como especialista en trasplante hepático de la Facultad de Medicina de la Universidad de São Paulo, Brasil, con desarrollo de estudios para optar por el título de doctor en trasplante uterino de la misma universidad, quien intervino en el desarrollo pre y posquirúrgico para el trasplante uterino en humanos que realizó el grupo profesional del Hospital Das Clínicas de la Facultad de Medicina de Ribeirão Preto, de la Universidad de São Paulo, Brasil; asimismo, se contó con la participación otros dos cirujanos de trasplante hepático con certificación formal, entrenados en Francia y Estados Unidos, quienes ejecutan cirugías de alta complejidad de hígado, vías biliares y páncreas; y dos médicos especialistas en ginecología y obstetricia, profesores universitarios. El equipo contó, además, con dos médicos veterinarios, un zootecnista especialista en especies menores, profesor del Servicio Nacional de Aprendizaje (SENA) de Tuluá; un profesor de farmacología y medicina regenerativa, y un bacteriólogo, pertenecientes al grupo profesional de la Facultad de Salud de la Universidad del Valle. Tamaño muestral: 8 ovejas.

Procedimiento. Las ovejas fueron evaluadas por un médico-veterinario y el zootecnista para verificar los criterios de selección. Se les realizó intervención quirúrgica consistente en explante y trasplante ortotópico de útero, incluyendo ovarios y tubas uterinas a través de una laparotomía: a continuación describimos el manejo preoperatorio e intraoperatorio de la oveja donante y en la oveja receptora el manejo posoperatorio y su seguimiento. 
A todas las ovejas se les efectuaron previamente pruebas de compatibilidad de los grupos-tipo sanguíneo con cruce de pruebas entre ellas, a fin de descartar familiaridad directa. La preparación prequirúrgica de cada animal consistió en mantenerlo en ayuno mínimo de 18 horas para alimentos sólidos, y un mínimo de 6 horas para la suspensión de la ingesta de líquidos (agua); en el periodo preanestésico todas las ovejas recibieron como medicamento sedativo y ansiolítico una dosis de midazolam intramuscular de $0,5 \mathrm{mg} / \mathrm{kg}$. Antes de la intervención se les administró profilaxis antibiótica con una única dosis de penicilina $\mathrm{G}$ procaínica (4.500.000 UI), penicilina G sódica (1.500.000 UI), sulfato de estreptomicina (7,5 g), acetonida de triancinolona (15 mg incluidos en una presentación comercial por 6.000.000 UI).

Descripción de la técnica y procedimiento en la oveja donante: el procedimiento anestésico fue realizado y supervisado por un médico veterinario con conocimiento en anestesiología veterinaria. Se realizó la inducción anestésica por vía endovenosa con propofol a una dosis de $5 \mathrm{mg} / \mathrm{kg}$ y, seguidamente, intubación orotraqueal, con tubo de tamaño apropiado $(7,5 \mathrm{u}$ $8 \mathrm{~mm}$ ). El mantenimiento anestésico se logró con isoflurano al 1,5\% en $100 \%$ de oxígeno, más una infusión endovenosa continua de fentanilo a 10 $\mathrm{ug} / \mathrm{kg} / \mathrm{min}$ y se les generó bloqueo neuromuscular con pancuronio a $0,05 \mathrm{mg} / \mathrm{kg}$, endovenoso; posteriormente se realizó intubación orotraqueal. Se mantuvo la anestesia con isoflurano 1,5\% en $100 \%$ de oxígeno y fentanilo endovenoso a $10 \mathrm{ug} / \mathrm{kg} / \mathrm{min}$. La capnografía se mantuvo entre 35 y $45 \mathrm{~mm} / \mathrm{Hg}$. Previo a realizar la incisión quirúrgica en cada animal ya anestesiado se efectuó lavado exhaustivo de la pared abdominal con jabón y solución de clorhexidina, para proceder seguidamente a realizar una vía de acceso quirúrgico abdominal por laparotomía mediana infraumbilical. Dada la limitación operativa de la canalización uretral por ser de calibre muy pequeño, se procedió a efectuar cateterismo o sondaje vesical por cistostomía, utilizando una sonda de Foley de tamaño 10-12F, la cual se dejó con drenaje continuo, cerrado. Se identificaron las estructuras viscerales y vasculares pélvicas. A continuación, se efectuó la ligadura, la reparación y la sección del ligamento redondo a nivel de su inserción pélvica, a fin de conservar su mayor longitud para lograr la sujeción del órgano trasplantado; se realizó disección de los vasos gonadales hasta su salida de los vasos iliacos y se hizo su reparación, también se reparó el uréter. Se procedió a realizar la disección y la reparación de la vena y arteria uterinas hasta su salida de la arteria iliaca interna, separándola del uréter; se repitieron los pasos del lado contralateral. Posteriormente, se realizó la identificación del cuello del útero y se disecó intraabdominalmente liberando la vagina hasta los dos tercios superiores, luego se pinzaron y seccionaron los vasos gonadales y uterinos, seguido de la sección de la vagina y el cierre de esta con sutura continua; se cerró la aponeurosis con polipropilene \# 1 y se suturó la piel con un material monofilamento absorbible. Al final del procedimiento de revirtió la anestesia con la administración de 0,02 mg/kg de flumazenil endovenoso.

Para el manejo del útero, las trompas y los ovarios extraídos se lavó el útero explantado bajo condiciones estrictas de antisepsia, en un bandeja estéril, con un litro de solución de lactato de Ringer y adición de 5.000 unidades de heparina, canalizando un vaso y verificando la permeabilidad de cada arteria uterina, efectuando irrigación intravascular; posteriormente, el órgano retirado fue sometido a hipotermia dejándose inmerso en solución salina normal al 0,9\% a una temperatura de $4^{\circ} \mathrm{C}$, se continuó con el procedimiento conocido como "cirugía de banco", en la cual se preparan los pedículos vasculares (2 arterias y 2 venas), a través de una disección minuciosa; seguidamente el órgano es perfundido con 1 litro de solución de lactato de Ringer, comprobando la salida del líquido por las venas gonadales. Se procedió luego a ocluir los vasos seccionados del plexo vascular inferior, y se evaluó nuevamente presencia del flujo de salida. Todos los órganos fueron conectados a una máquina de 


\section{Figura 1.}

Aspecto del útero de oveja para ser y trasplantado posterior a la cirugía de banco en trasplante uterino en oveja, Cali, Colombia, 2018

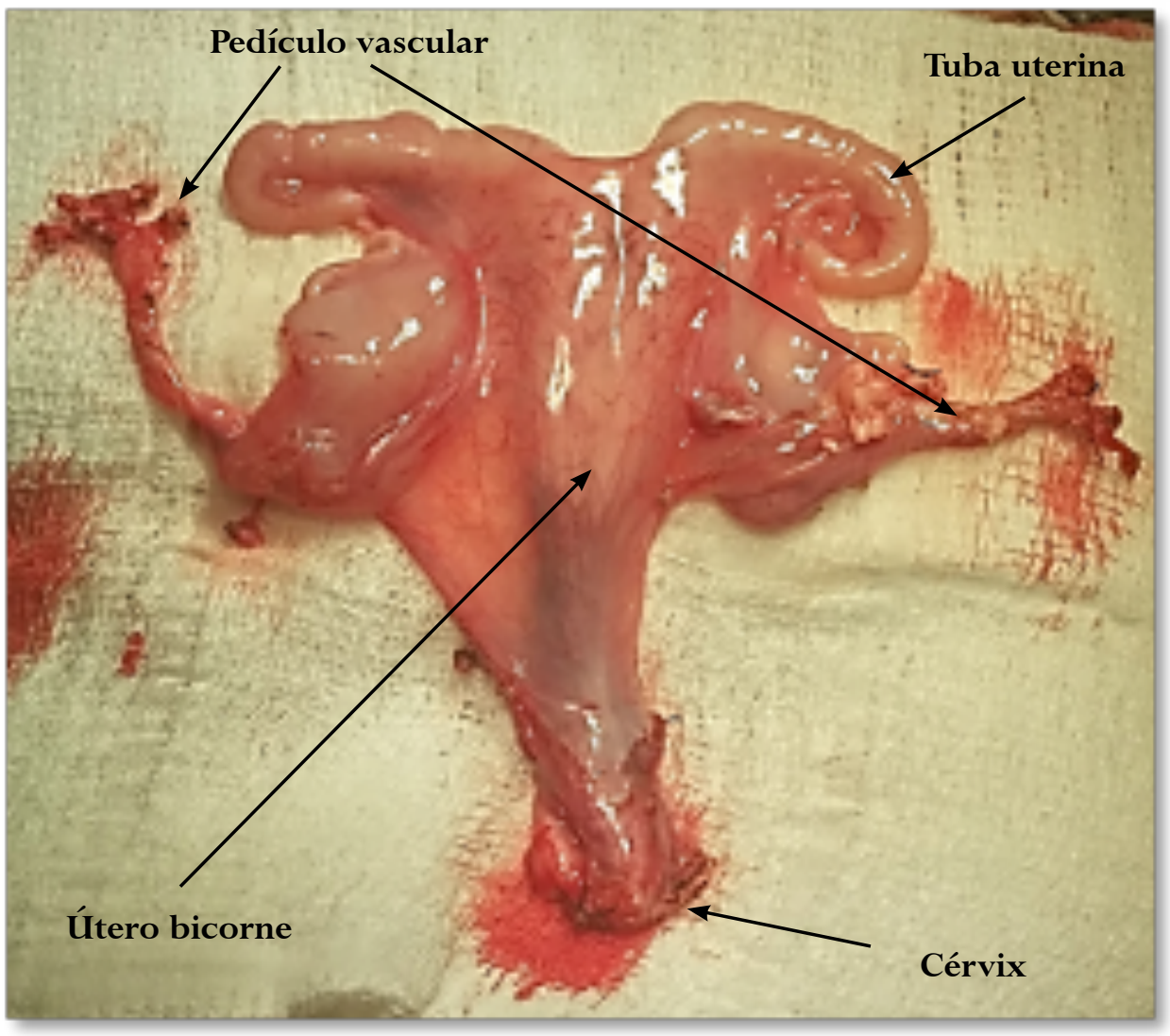

perfusión continua, irrigándose solución a través de las arterias gonadales, posterior a la ejecución de la cirugía de banco (figura 1) y manteniendo condiciones de hipotérmica entre 4 a $6^{\circ} \mathrm{C}$, usándose una solución isotónica por 20 a 24 horas. La máquina de perfusión continua fue diseñada por profesores de ciencias básicas de la Facultad de Salud de la Universidad del Valle.

Cirugía en la oveja receptora: previo a la cirugía del implante, los úteros se mantuvieron inmersos y perfundidos con una solución normotérmica a temperatura constante de $37^{\circ} \mathrm{C}$. Al final del procedimiento descrito, para la exéresis de útero, vagina, trompas y ovarios, a cuatro de las ovejas se les realizó trasplante de útero; para esto, se dejó la porción residual de la vagina reparada con puntos de sutura usando poliglactina 910, de calibre \# 1, separando los ángulos; posteriormente, se procedió a disecar proximalmente la arteria y la vena iliaca externas de ambos lados y se colocó el injerto (útero) en la cavidad pélvica. Se inyectaron $20 \mathrm{~mL}$ de suero heparinizado (preparado con $100 \mathrm{~mL}$ de solución salina y 5.000 unidades de heparina) por cada arteria uterina y se realizó la anastomosis términolateral de la arteria gonadal del injerto con la arteria iliaca externa de la receptora usando polipropilene \# 8-0. Posteriormente a la anastomosis arterial, se realizó la anastomosis venosa ipsilateral con técnica término-lateral con la vena iliaca externa, usando polipropilene de calibre \# 5-0. Una vez finalizadas las dos primeras anastomosis vasculares, se procedió a permitir el paso sanguíneo con el propósito de 
facilitar la rápida perfusión del órgano trasplantado. A continuación, se repitieron los mismos pasos del lado contralateral y, seguidamente, se realizó una revisión exhaustiva de las anastomosis en los vasos gonadales una a una, para evaluar la presencia de posibles fugas vasculares. Aquellos vasos que no pudieron ser recanalizados fueron ligados selectivamente; en todos los casos se logró más de una anastomosis de arteria y vena bilateralmente. Después se procedió a la anastomosis vaginal, término-terminal con sutura continua de poliglactina 910 de calibre \# 1, y se continuó con la fijación del útero con los remanentes de los ligamentos redondos fijándolos a la pared pélvica en ambos lados, usando como material de sutura poliglactina 910 de calibre \# 1; para finalizar se verificó una vez más la hemostasia en la cavidad abdomino-pélvica y se procedió luego al retiro de la sonda Foley en vejiga, realizándose seguidamente cistorrafia. Como parte final se realizó el cierre de la aponeurosis con polipropilene de calibre \# 1 y cierre de la piel con poliglecaprone 25 de calibre \# 3-0.

El manejo posoperatorio de las ovejas estuvo bajo el cuidado de médico veterinario y zootecnólogo, en un criadero especializado de ovejas. Todas las ovejas trasplantadas recibieron 40 unidades de enoxaparina diarias vía subcutánea, durante la primera semana del posoperatorio.

Seguimiento: a todos los animales trasplantados se les realiza una evaluación directa del cérvix uterino a través de especuloscopias directas, en los días 7, 15, 30 y 180 después de la cirugía del trasplante. Adicionalmente, los médicos-veterinarios les realizaron estudios ultrasonográficos transabdominales y endorrectales cada 3 meses.

Variables medidas: edad y peso de las ovejas, tiempo quirúrgico, tiempo de isquemia fría y caliente, sangrado y complicaciones intraoperatorias, efectividad determinada por el logro de anastomosis vasculares funcionales y conservación de la vitalidad a corto tiempo en los animales intervenidos con trasplante de útero, la seguridad por la presencia de infección posoperatoria, sangrado posoperatorio, rechazo del injerto, mortalidad asociada al procedimiento. Se evaluó la morfología del útero y ovarios por ecografía a los 15, 30 y 180 días.

Análisis estadístico. Se hace un análisis descriptivo, la información de las variables continuas se presenta con las medianas y los rangos interquartiles (RIQ), y las variables categóricas se presentan como proporciones.

Aspectos éticos. Este estudio contó con la aprobación del Comité Institucional de Revisión de Ética con Animales en Experimentación de la Facultad de Salud de la Universidad del Valle, aprobado el 26 de septiembre de 2017, código interno 011-017, llevando en consideración los tiempos quirúrgicos como parámetro de evolución técnica y el cuidado posoperatorio del animal, con seguimiento y evaluación veterinaria.

\section{RESULTADOS}

En el periodo de tiempo descrito fueron intervenidas 8 ovejas. Un total de 4 ovejas fueron explantadas y sirvieron como donantes, y 4 ovejas fueron seleccionadas como receptoras, en quienes además de ser histerectomizadas, posteriormente se les trasplantaron el útero, las tubas y los ovarios de otras ovejas.

La mediana del peso de las ovejas donantes fue de $42 \mathrm{~kg}$ (rango: 41 - 47), y en las ovejas receptoras fue de 41,5 kg; (rango: 40 - 44); los animales eran de diferentes razas, no compatibles en la evaluación de la reacción cruzada de los grupos sanguíneos, a fin de eliminar el cruce entre animales de un mismo grupo familiar. La mediana del tiempo de isquemia caliente fue de 35,5 minutos, con rango intercuartil [RIQ]: 28,5-40, la mediana del tiempo quirúrgico de las cirugías de trasplante fue de 218,5 minutos (RIQ: 198,5-232,5); el tiempo total de la cirugía de trasplante uterino disminuyó progresivamente de 240 minutos para el primer procedimiento, hasta un tiempo de 185 minutos en la última intervención, mientras que la evaluación del tiempo de isquemia caliente para cada útero por trasplantar se redujo de 42 a 22 minutos (figura 2). 
Figura 2.

Tiempo de isquemia caliente y duración de los procedimientos en trasplante uterino en Ovejas, Cali, Colombia, 2018

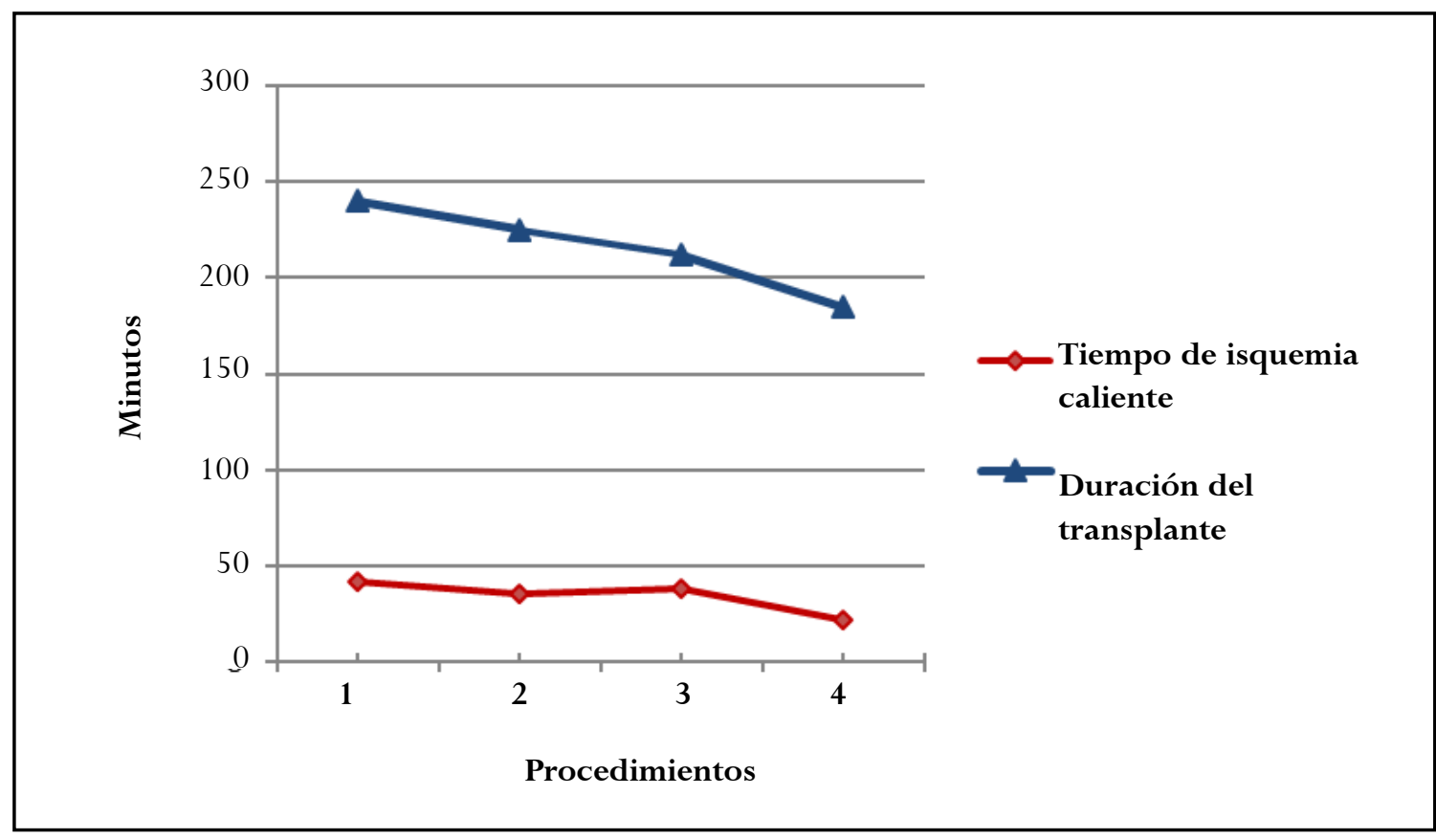

Se observa disminución de los tiempos de duración de la cirugía al igual que los tiempos de isquemia caliente. A medida que se avanzó en los procedimientos se adquirió mayor destreza.

No se presentaron cirugías fallidas en los procedimientos de explante, como tampoco en los de trasplante. La primera oveja trasplantada falleció al séptimo día posoperatorio, y le fue realizada la necropsia por médico veterinario, quien evidenció una obstrucción y trombosis a nivel intestinal, secundaria a la presencia intraabdominal de un cuerpo extraño (compresa), fenómeno trombótico que se definió como causa de muerte; adicionalmente, se encontró que el aspecto macroscópico del útero y los ovarios era homogéneo, sin evidencia de edema ni áreas de necrosis, y las anastomosis, al igual que los pedículos vasculares tanto arteriales como venosos de ambos lados estaban permeables, sin signos macroscópicos de isquemia. En la segunda oveja trasplantada no hubo complicaciones intraoperatorias o tempranas, se identificó infección de la cavidad vaginal durante la realización de la especuloscopia al séptimo día posquirúrgico (se observó palidez del cérvix uterino y presencia de flujo verde, fétido), se le dio tratamiento con metronidazol aplicado vía intravaginal, una vez al día, durante 7 días continuos, con evolución favorable en términos de desaparición de la infección y mejoría macroscópica del aspecto del cérvix uterino.

La tercera y cuarta ovejas trasplantadas evolucionaron satisfactoriamente. Las tres ovejas que sobrevivieron al procedimiento del trasplante se encontraban bien los días 15, 30 y 180 del posoperatorio. Hasta ese momento del seguimiento no se había logrado ningún embarazo espontáneo.

\section{DISCUSIÓN}

Presentamos los resultados de cirugía experimental con ovejas, efectuando explante e implante de útero, tubas y ovarios. No se tuvieron procedimientos fallidos en relación con la cirugía de explante y trasplante del útero, se presentaron complicaciones 
posoperatorias en 2 ovejas, una de las cuales fallece y otra presenta infección vaginal no grave. En términos de sobrevida de la cirugía de trasplante, 3 ovejas se encontraban en buen estado a los 6 meses. No se ha logrado embarazo espontáneo en estas ovejas un año después. Este trabajo permitió el entrenamiento en técnica quirúrgica a un equipo de cirujanos colombianos en trasplante uterino. Nuestro estudio empleó la técnica quirúrgica utilizada en la cirugía de banco, necesaria para la preparación de los pedículos vasculares del injerto antes de su trasplante, descrita por otros autores (19-21).

Se logró una disminución de 4 a 3 horas del tiempo intraquirúrgico tanto para la cirugía de extracción como para la posterior colocación del órgano-injerto; de igual manera, se obtuvo resultado con acortamiento en los tiempos de intervención en la fase de isquemia caliente, evidenciada de manera notoria y progresiva en los últimos procedimientos efectuados. En cuanto a la duración de la cirugía Brännström et al. describen que este tiempo varió entre 3 y 4 horas (22).

Una limitación de este estudio es que no ha sido posible la realización de controles sanguíneos de niveles hormonales en las ovejas, proceso muy complejo para su procesamiento en Colombia, por términos del aval técnico correspondiente para los laboratorios veterinarios en la región; aun así, el modelo ovino presenta más facilidades para su intervención en términos de consecución y el cuidado de los animales en todas las fases: antes, durante y después de la cirugía de trasplante uterino. Algunos autores describen que el modelo en primates no humanos es más adecuado por tener ciclos menstruales parecidos a los del humano (23) y que la perfusión del útero se hace principalmente por las arterias uterinas y las arterias ováricas y vaginales, como también el retorno venoso principalmente a través de las venas ováricas (9). Por otra parte, no se realizaron estimulaciones hormonales farmacológicas a las ovejas trasplantadas, ni se implementó un plan de tratamiento con métodos de reproducción asistida para lograr embarazos, dado que no se habían planteado como objetivos del estudio.

\section{CONCLUSIÓN}

El modelo ovino permite el entrenamiento para grupos médico-quirúrgicos que desean hacer cirugía experimental de trasplante uterino en animales. Esta experiencia constituyó para los autores un paso trascendental en la adquisición de conocimiento que permitirá a investigadores de la Universidad del Valle y del Centro Médico Imbanaco avanzar hacia la realización de este tipo de trasplante en mujeres con infertilidad por factor uterino.

\section{FINANCIACIÓN}

La financiación del estudio provino del Centro Médico Imbanaco de Cali S.A., de la Facultad de Salud de la Universidad del Valle y una parte a través de recursos propios de los autores.

\section{AGRADECIMIENTOS}

Los autores expresan sus agradecimientos a los profesionales que contribuyeron al desarrollo de este estudio: Jaime Muñoz Botina, bacteriólogo funcionario del Laboratorio de Farmacología y Medicina Regenerativa de la Facultad de Salud de la Universidad del Valle; Rodrigo Zambrano Galeano, médico veterinario; Luz Adriana Ramírez Luna, médica veterinaria e instructora del SENA-Tuluá; Gustavo Adolfo Rivera Cruz, zootecnólogo instructor en producción de especies menores del SENATuluá; Angie Londoño Betancur, instrumentadora quirúrgica y Lizbeth Lenis, auxiliar de enfermería, circulante de quirófanos, pertenecientes al Centro Médico Imbanaco de Cali S.A.

\section{REFERENCIAS}

1. World Health Organization - Human Reproduction Programme. Sexual and reproductive health. Infertility definitions and terminology. WHO-HRP; 2016. Disponible en: https://www.who.int/reproductivehealth/ topics/infertility/definitions/en/ 
2. Ministerio de Salud/Profamilia. Encuesta Nacional de Demografía y Salud. Colombia. 2015. Disponible en: https://dhsprogram.com/pubs/pdf/FR334/FR334.pdf

3. Mascarenhas MN, Flaxman SR, Boerma T, Vanderpoel S, Stevens GA. National, regional, and global trends in infertility prevalence since 1990: A systematic analysis of 277 health surveys. PLoS Med. 2012;9(12):e1001356. https://doi.org/10.1371/ journal.pmed.1001356

4. Taylor E, Gomel V. The uterus and fertility. Fertil Steril. 2008;89(1):1-16. https://doi.org/10.1016/j. fertnstert.2007.09.069

5. Acién P. Incidence of Müllerian defects in fertile and infertile women. Hum Reprod. 1997;12(7):13726. https://doi.org/10.1093/oxfordjournals.humrep. a019588

6. Monteiro CS, Cavallo IK, Dias JA, Pereira FAN, Reis FM. Uterine alterations in women undergoing routine hysteroscopy before in vitro fertilization: High prevalence of unsuspected lesions. JBRA Assist Reprod. 2019;23(4):396-401. https://doi.org/10.5935/15180557.20190046

7. Raga F, Bauset C, Remohi J, Bonilla-Musoles F, Simon C, Pellicer A. Reproductive impact of congenital Mullerian anomalies. Hum Reprod. 1997;12(10):227781. https://doi.org/10.1093/humrep/12.10.2277

8. American Fertility Society. Classification of adnexal adhesions, distal tubal occlusion, tubal occlusion secondary to tubal ligation, tubal pregnancies, mullerian anomalies and intrauterine adhesions. Fertil Steril. 1988;49(6):944-55. https://doi.org/10.1016/ s0015-0282(16)59942-7

9. Johannesson L, Dahm-Kähler P, Eklind S, Brännström M. The future of human uterus transplantation. Womens Health. 2014;10(4):455-67. https://doi. org/10.2217/WHE.14.22

10. Corte Constitucional, Sentencia T-226/10 de 23 de marzo de 2010. Magistrado Ponente: Mauricio González Cuervo. Disponible en: http:/www.corteconstitucional.gov.co/RELATORIA/2010/T-226-10. htm

11. Beetar Bechara B. La maternidad subrogada en Colombia: hacia un marco jurídico integral e incluyente.
Estud Soc. 2019;21(2). https://doi.org/10.12804/ revistas.urosario.edu.co/sociojuridicos/a.6869

12. Fageeh W, Raffa H, Jabbad H, Marzouki A. Transplantation of the human uterus. Int J Gynaecol Obstet. 2002;76(3):245-51. https://doi.org/10.1016/S00207292(01)00597-5

13. Brännström M, Johannesson L, Bokström H, Kvarnström N, Mölne J, Dahm-Kähler P, et al. Livebirth after uterus transplantation. Lancet. 2015;385:607-16. https://doi.org/10.1016/S0140-6736(14)61728-1

14. Ejzenberg D, Mendes LR, Haddad LB, Baracat EC, D’Albuquerque LA, Andraus W. Uterine transplantation: A systematic review. Clinics (São Paulo). 2016;71(11): 679-83. https://doi.org/10.6061/clinics/2016(11)10

15. O'Donovan L, Williams NJ, Wilkinson S. Ethical and policy issues raised by uterus transplants. Br Med Bull. 2019;131(1):19-28. https://doi.org/10.1093/bmb/ ldz022

16. Milliez J. Uterine transplantation FIGO Committee for the ethical aspects of human reproduction and women's health. Int J Gynaecol Obstet. 2009;106(3):270. https://doi.org/10.1016/j.ijgo.2009.03.045.

17. Eraslan S, Hamernik RJ, Hardy JD. Replantation of uterus and ovaries in dogs, with successful pregnancy. Arch Surg. 1966;92(1):9-12. https://doi.org/10.1001/ archsurg.1966.01320190011002

18. Tzakis AG. Nonhuman primates as models for transplantation of the uterus. Fertil Steril. 2013;100(1):61. https://doi.org/10.1016/j.fertnstert.2013.03.004

19. Andraus W, Ejzenberg D, Santos RM, et al. Sheep model for uterine transplantation: The best option before starting a human program. Clinics (São Paulo). 2017;72(3):178-82. https://doi.org/10.6061/clinics/2017(03)08

20. Dahm-Kähler P, Wranning C, Lundmark C, et al. Transplantation of the uterus in sheep: Methodology and early reperfusion events. J Obstet Gynaecol Res. 2008;34(5):784-93. https://doi.org/10.1111/j.14470756.2008.00854.x

21. Wranning CA, Marcickiewicz J, Enskog A, DahmKähler P, Hanafy A, Brännström M. Fertility after autologous ovine uterine-tubal-ovarian transplantation by vascular anastomosis to the external iliac 
vessels. Hum Reprod. 2010;25(8):1973-9. https:// doi.org/10.1093/humrep/deq130

22. Brännström M, Wranning CA, Altchek A. Experimental uterus transplantation. Hum Reprod Update. 2010;16(3):329-45. https://doi.org/10.1093/ humupd/dmp049

23. Martin LJ, Carey KD, Comuzzie AG. Variation in menstrual cycle length and cessation of menstruation in captive raised baboons. Mech Ageing Dev. 2003;124(8-9),865-71. https://doi.org/10.1016/ S0047-6374(03)00134-9

\section{CONTRIBUCIONES DE LOS AUTORES}

Felipe Castro-Villegas: concepción y diseño del estudio, participación como cirujano en las intervenciones quirúrgicas de las ovejas, responsable del análisis y revisión final del manuscrito.
Gustavo Canaval-Erazo: concepción y diseño del estudio, participación como cirujano en las intervenciones quirúrgicas de las ovejas, responsable del análisis y revisión final del manuscrito.

Juan Manuel Rico-Juri: participación como cirujano en las intervenciones quirúrgicas de las ovejas.

José Óscar Gutiérrez-Montes: participación en las preparaciones y adecuaciones de mantenimiento a flujo continuo de los órganos explantados, así como el análisis de los exámenes de compatibilidad.

Anabel Vanin-Aguas: participación como cirujano en las intervenciones quirúrgicas de las ovejas.

Hoover Canaval-Erazo: concepción y diseño del estudio, participación como cirujano en las intervenciones quirúrgicas de las ovejas, responsable del análisis y revisión final del manuscrito. 